\title{
Development of Spinal Neurons and Tracts in the Zebrafish Embryo
}

\author{
JOHN Y. KUWADA, ROBERT R. BERNHARDT, AND NGUYEN NGUYEN \\ Department of Biology (J.Y.K., R.R.B., N.N.), Institute of Gerontology (J.Y.K.), and the \\ Neuroscience Program (J.Y.K.), University of Michigan, Ann Arbor, Michigan 48109
}

\begin{abstract}
We have analyzed pathfinding by growth cones in the spinal cord of the early zebrafish embryo, because it is an extremely simple system. At 18-20 hours of development the spinal cord contains approximately 18 lateral and presumably post-mitotic cell bodies per hemisegment. Of these 8-11 have projected growth cones by $18 \mathrm{hr}$ of development and fall into five classes of neurons (Bernhardt et al., J. Comp. Neurol, preceding paper), including a set of mechanosensory (RB) neurons, three classes of interneurons (DoLA, ascending commissural, and VeLD), and previously characterized primary motor neurons (Eisen et al., '86: Nature 320:269-271). Of these five classes we analyzed pathfinding by the RB, DoLA, early ascending commissural, and VeLD neurons. These neurons are distinguishable at the earliest stages of axonogenesis based on the location of their somata and the number and initial directionality of their growth cones. In each case they follow stereotyped, cell-specific pathways to reach their termination sites. Up through larval stages exuberant axons have not been observed.

The longitudinal axons of each neuronal class form bundles in the early cord. This apparently occurs because growth cones extend in close association with the longitudinal axons of the same neuronal class. At later stages spatially discrete commissural tracts are found in the cord suggesting that commissural growth cones may follow earlier commissural axons as well.
\end{abstract}

Key words: growth cones, pathfinding, embryonic spinal cord

The spinal cord of the early zebrafish embryo is a simple system containing a small number of neurons. At $18-20$ hours in development each hemisegment contains approximately 18 lateral and presumably post-mitotic cells. Of these, the eight to eleven earliest neurons to project growth cones fall into five classes. These are mechanosensory neurons, Rohon-Beard (RB) cells; dorsal longitudinal ascending (DoLA), ventral longitudinal descending (VeLD), and ascending commissural interneurons (Bernhardt et al., '90); and primary motor neurons (Myers et al., '86). The simplicity of the zebrafish embryonic cord makes it an attractive system for analysis of pathfinding by growth cones in the vertebrate central nervous system (CNS).

Previous studies have demonstrated that in zebrafish embryos the primary motor neurons (Eisen et al., '86) and one cluster of brain neurons (Chitnis and Kuwada, '90) project growth cones which follow cell-specific pathways to reach their targets. Similar findings have been made for several classes of spinal neurons in the embryos of the Japanese medaka fish (Kuwada, '86). Here we describe the pattern of axonogenesis by four of the earliest classes of spinal neurons in the zebrafish embryos and the development of the axonal tracts in the spinal cord.

\section{MATERIALS AND METHODS}

Procedures for labeling embryonic neurons are given in the companion paper (Bernhardt et al., '90). In some cases diI labeled neurons were marked with a dark reaction product by following the photooxidation procedure (Maranto, '83; Sandell and Masland, '88). Embryos were prepared for electron microscopy (EM) by fixing in $3 \%$ glutaraldehyde, $2 \%$ paraformaldehyde, $1 \%$ acrolein, and $1 \%$ DMSO in $75 \mathrm{mM}$ phosphate buffer for 1 hour, washed, postfixed in $2 \%$ osmium tetroxide in phosphate buffer for 2 hours, washed in $50 \mathrm{mM}$ sodium maleate buffer ( $\mathrm{pH} 5.9$ ), stained with $2 \%$ uranyl acetate in maleate buffer for 1-2 hours, washed in maleate buffer, dehydrated in an ethanol and propylene oxide series, embedded in plastic, and thin sectioned.

\section{RESULTS \\ Pathfinding by the earliest spinal growth cones}

All four of the earliest neuronal classes studied projected the appropriate number of growth cones at 15-17 hours of

Accepted August 30, 1990. 

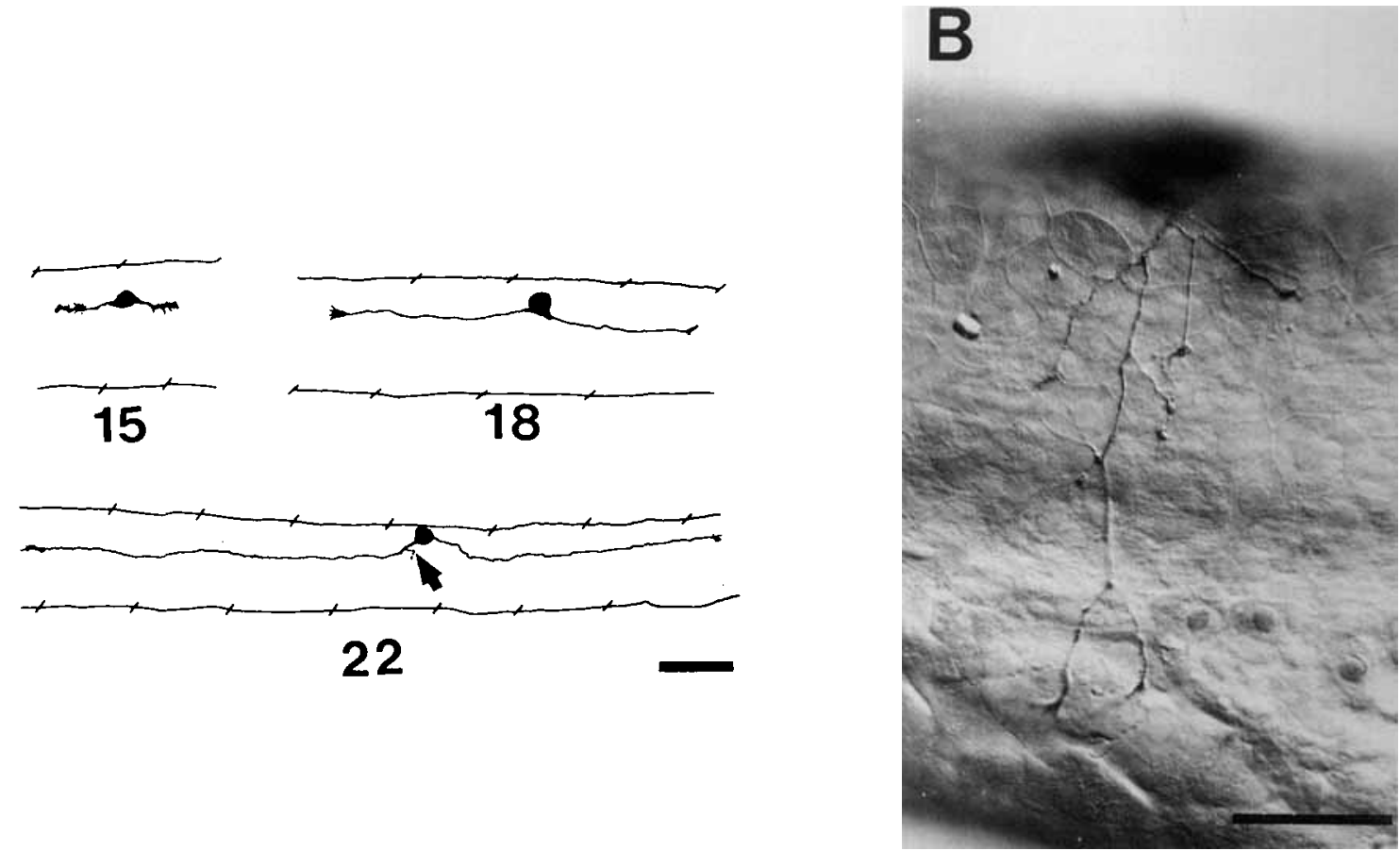

Fig. 1. Development of RB neurons. A: Camera lucida drawings of LY-filled RB neurons at various stages in development. This and all other figures are sideviews with anterior to the left, dorsal up, the dorsal and ventral boundaries of the cord outlined, and diagonal lines

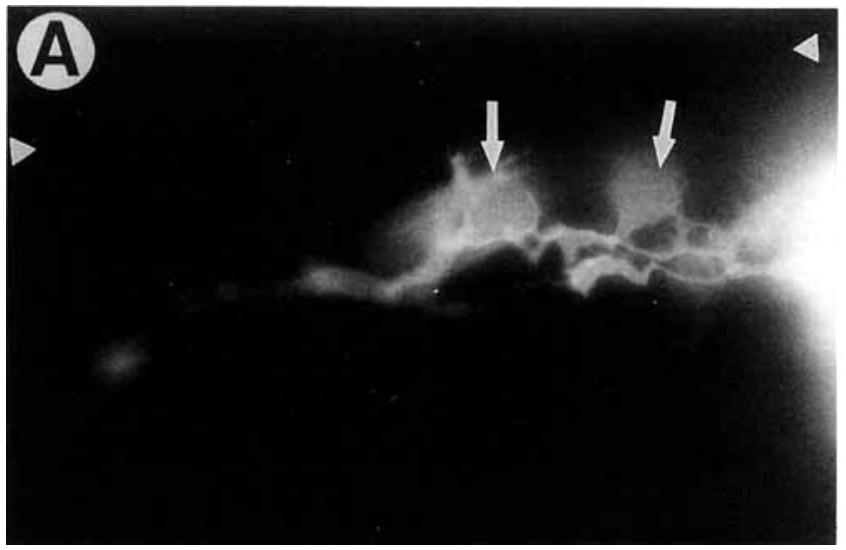

Fig. 2. RB neurons pioneer the DLF. Triangles denote the dorsal border of the cord. A: Micrograph showing that only RB neurons (arrows) are backlabeled by an application of diI (seen in the right side of the photograph) to the dorsal cord in a 16-17-hour embryo. B:

\section{Abbreviations}

CON1 carp optic nerve 1

dil $1^{\prime}-1^{\prime}$-dioctadecyl-3-3-3'-3'-tetramethylindocarbocyanine perchlorate

DLF dorsal longitudinal fasciculus

DoLA dorsal longitudinal ascending

HRP horseradish peroxidase

MLF medial longitudinal fasciculus

LY lucifer yellow

RB Rohon-Beard

VLF ventral longitudinal fasciculus representing segment boundaries unless stated otherwise. Only the proximal portion of the peripheral axon (arrow) has been drawn. Scale, $50 \mu \mathrm{m}$. B: Micrograph of the peripheral axons of a LY/anti-LY-labeled $\mathrm{RB}$ cell in the skin of a 21 -hour embryo. Scale, $50 \mu \mathrm{m}$.

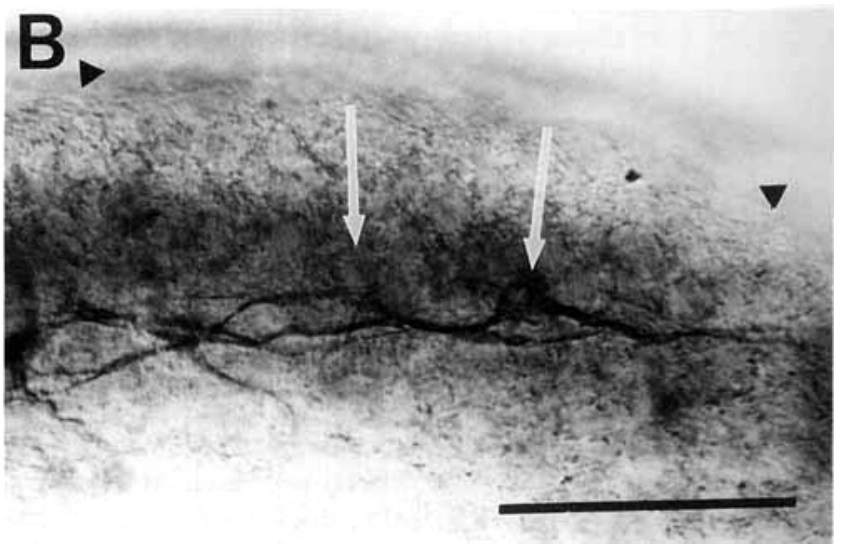

Micrograph of acetylated tubulin-labeled cord showing that $\mathrm{RB}$ axons make up the DLF at 16 hours. Weakly labeled RB cell bodies are indicated by arrows. Scale for A and B, $50 \mu \mathrm{m}$.

development. These include the RB, DoLA, early ascending commissural, and VeLD neurons (see below). Although the earliest members of each of these neuronal classes project growth cones during this period, other members of these classes often begin axonogenesis later. This is especially apparent for the $\mathrm{RB}$ and ascending commissural neurons (Bernhardt, et al., '90). Nevertheless, in each case these growth cones extended along stereotyped, cell-specific pathways to their normal termination sites in the CNS. 


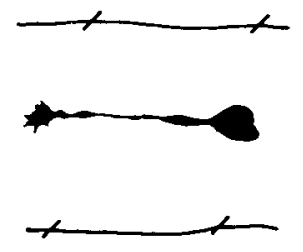

18

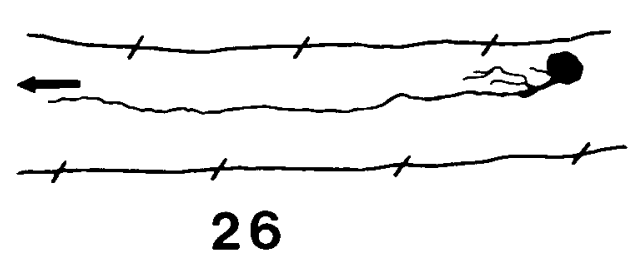

Fig. 3. Development of DoLA neurons. Camera lucida drawings of LY-filled DoLA neurons at various stages in development. Arrows denote that the axons extend beyond the drawing. At 26 hours the axon terminated in the hindbrain near the otocyst. Note the ventrally directed processes at 42 hours. Scale, 50 $\mu \mathrm{m}$.
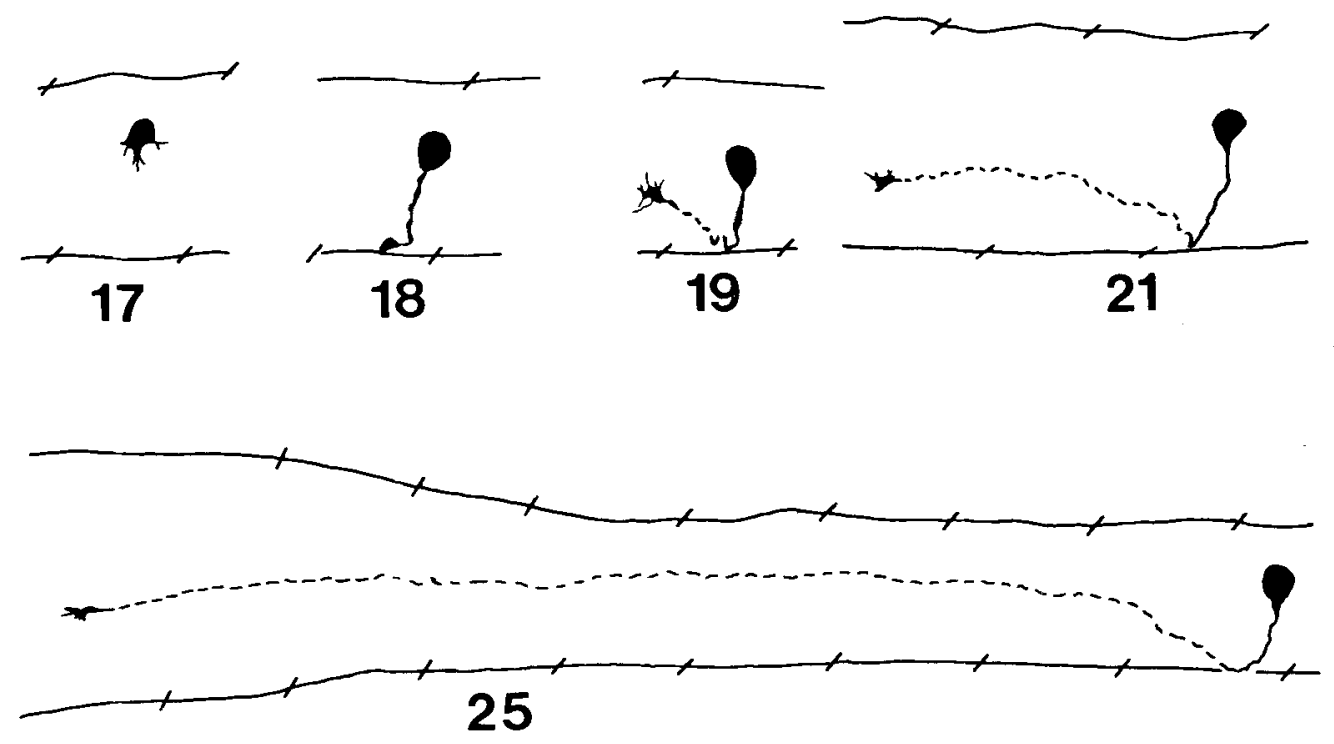

Fig. 4. Development of early differentiating ascending commissural neurons. Camera lucida drawings of LY-filled neurons at various stages in development. In this and all subsequent figures the axon contralateral to the cell body is dashed. Contralateral growth cones are not dashed. Scale, $50 \mu \mathrm{m}$.

Axonogenesis by these neurons has been reconstructed from 467 intracellular fills of identified neurons with Lucifer Yellow (LY) dye in 211 embryos between 15 and 48 hours of development from segments 5-15. These include 51 RB, 82 DoLA, 200 early ascending commissural, and 134
VeLD neurons. Of these, 112 neurons were filled between 15 and 18 hours of development. Immature neurons were identified on the basis of their cell body location (Bernhardt et al., '90) and the number and initial directionality of their growth cones (see below). The spinal interneurons did not 


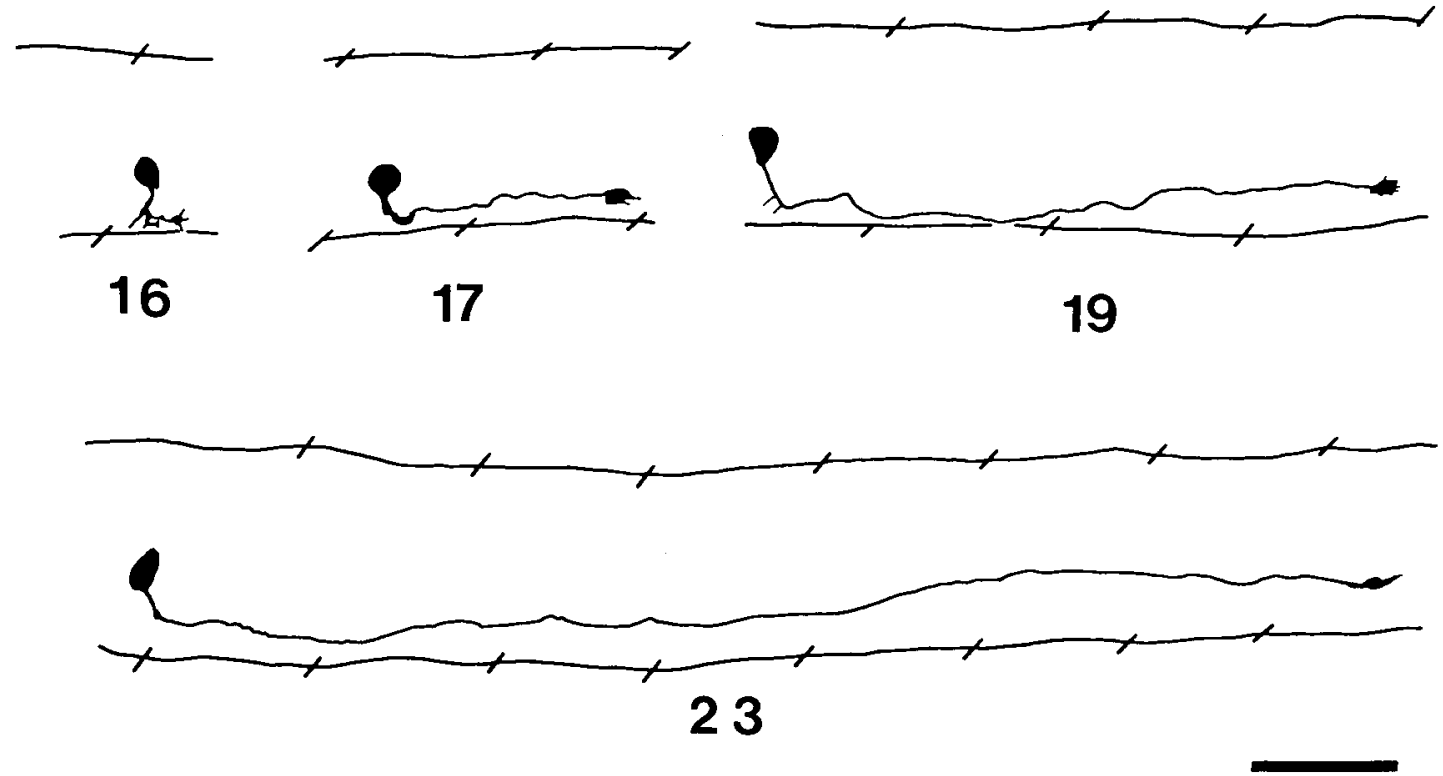

Fig. 5. Development of VeLD neurons. Camera lucida drawings of LY-filled VeLD neurons at various stages in development. Note that the growth cone is initially projected from the ventral pole of the soma. Scale, $50 \mu \mathrm{m}$.

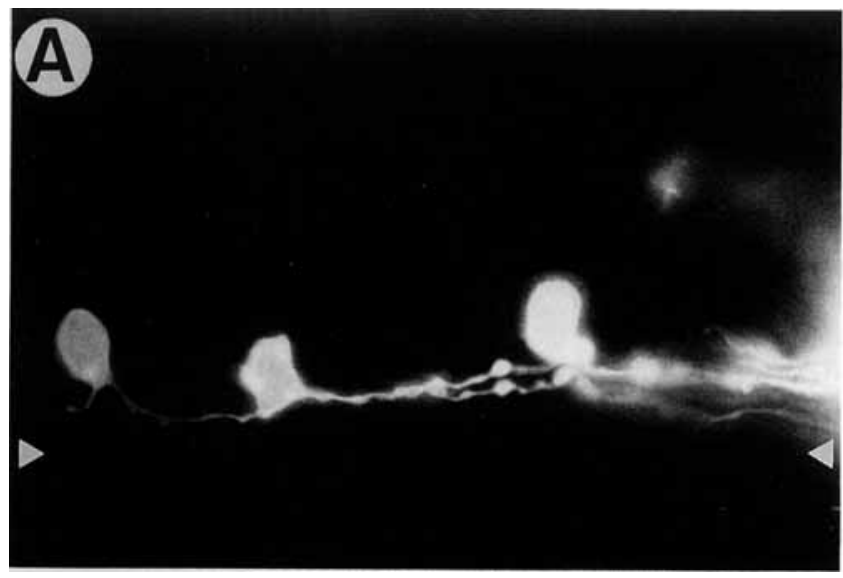

Fig. 6. VeLD neurons pioneer the VLF. Triangles denote the ventral border of the cord. A: Micrograph showing that only VeLD neurons are backlabeled by an application of diI to the ventral cord in a 17-hour embryo. B: Micrograph showing the VeLD axons make up the VLF in

display any obvious gradient of development along the anterior/posterior axis in midbody segments 5-10 (data not shown) much like zebrafish motor neurons (Myers et al., '86) although differences were obvious when anterior segments were compared with segments posterior to the midbody segments.

$\boldsymbol{R B}$ neurons. The $\mathrm{RB}$ neurons appear to begin axonogenesis over an extended period of development (Bernhardt, et al., '90). The following description of axonogenesis applies to the earliest RB neurons to initiate axonogenesis. The earliest growth cones are projected by the $\mathrm{RB}$ neurons at 15 hours of development (Fig. 1). The RB neurons are located in the dorsolateral cord and project two growth

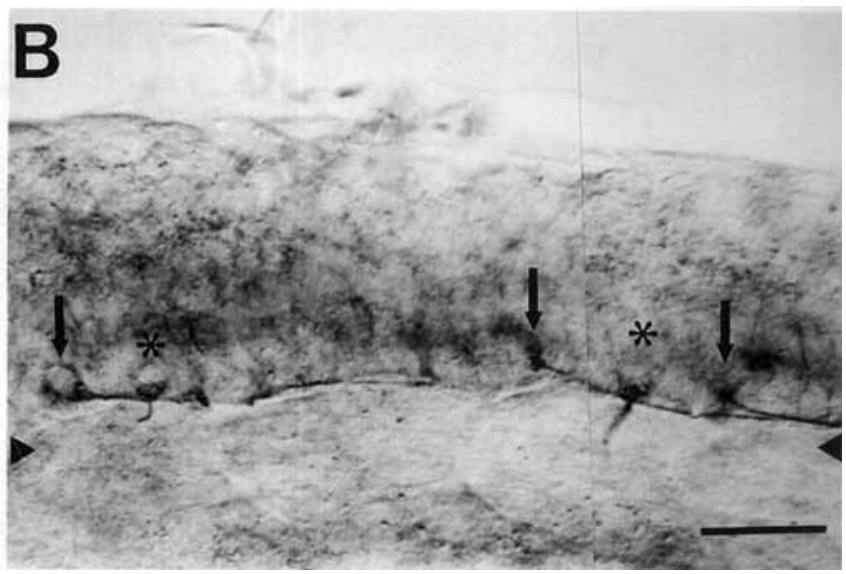

17-hour cords labeled with the acetylated tubulin antibody. VeLD cell bodies, arrows; primary motor neurons, asterisks; scale for A and B, 25 $\mu \mathrm{m}$.

cones, one rostrally and the other caudally. Each RB growth cone extends longitudinally in the dorsolateral region of the cord. Two observations indicate that the RB growth cones pioneer the dorsal longitudinal fasciculus (DLF). 1) Applications of diI to the dorsolateral cord in 16-17 hours embryos ( $\mathrm{n}=12$ ) backfilled only RB neurons (Fig. 2A). 2) A monoclonal antibody against acetylated tubulin (Piperno and Fuller, '85), which labels many if not all neurons which have begun axonogenesis (Chitnis and Kuwada, '90), labels only the RB neurons in the dorsolateral cord of $16 \mathrm{hr}$ embryos ( $n=12$; Fig. 2B). As early as 17 hours, but usually later, the peripheral axon projects from the longitudinal axon, usually within a segment from the cell body, to 

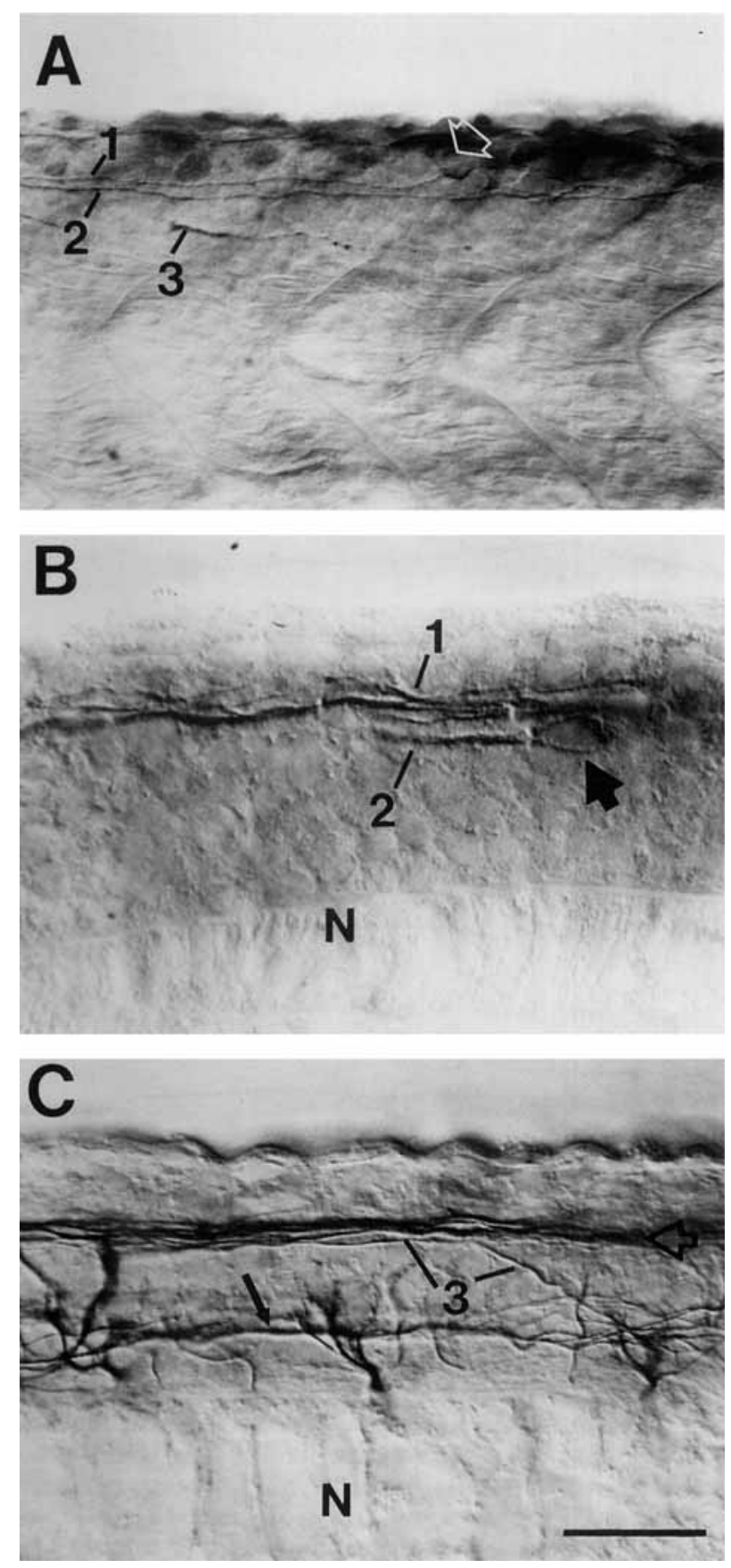

Fig. 7. Spatial organization of DLF axons. A: Micrograph showing that the LY/anti-LY-labeled Iongitudinal axon of the RB neuron (1), DoLA longitudinal axon (2), and the ascending commissural growth cone (3) are ordered from dorsal to ventral in the DLF in a 24-hour embryo. Each chevron-shaped outline is a single segment of axial muscles. Arrow, cell body of the labeled RB. B: Micrograph showing that the RB axons (1) are dorsal to a DoLA axon (2) and cell body (arrow) in a $18 \mathrm{hr}$ embryo labeled with the acetylated tubulin antibody. N, notochord. C: Micrograph showing that the longitudinal axon (3) of an ascending commissural neuron is ventral to the other longitudinal axons in the DLF (open arrow) labeled with the acetylated tubulin antibody in another 24-hour embryo. The cell body of the ascending commissural neuron is out of the focal plane on the contralateral side. Motor neurons and their axons and the VLF (arrow) are also labeled. Scale for A, $50 \mu \mathrm{m}$; scale for B and C, $25 \mu \mathrm{m}$. arborize in the skin. Eventually the RB axons terminate near the otocyst in the hindbrain and in the caudal end of the cord (Bernhardt et al., '90).

DoLA neuron. The DoLA interneuron is located ventral to the $\mathrm{RB}$ cells in the dorsolateral cord and projects a single growth cone at 17-18 hours of development (Fig. 3). This growth cone extends rostrally in the DLF. By $26 \mathrm{hr}$ some DoLA growth cones have reached the level of the otocyst in the hindbrain. The DoLA neuron projects numerous collateral longitudinal branches from the main axon close to the cell body in the later stages of development. Additionally, ventrally directed branches extending from the main axon into the ventral portion of the cord are evident by 42 hours.

Ascending commissural neuron. The larval cord contains two subclasses of ascending commissural neurons, commissural primary ascending (CoPA) and commissural secondary ascending (CoSA), and the earliest ascending commissural neuron to differentiate may be the CoPA neuron (Bernhardt et al., '90). The ascending commissural interneurons are located ventral to the $\mathrm{RB}$ cells in the dorsolateral cord and the earliest ones project growth cones from the ventral poles of their cell bodies as early as 16-17 hours of development. The growth cones subsequently make two directional changes during axonogenesis (Fig. 4). After emerging from the cell body, the growth cones extend ventrally along a circumferential route to reach the ventral midline by 18-19 hours. Upon crossing the midline the growth cones turn in a rostral direction and begin to extend in an oblique rostrodorsal pathway. By 20-21 hours they reach the level of the commissural cell bodies and turn to follow an ascending longitudinal pathway in the DLF. The growth cones then extend longitudinally to reach the level of the otocyst in the hindbrain by 27 hours.

VeLD neuron. The VeLD interneuron is located dorsal to the primary motor neurons in the ventrolateral cord and projects a single growth cone from the ventral pole of its cell body at 16-17 hours as does the ascending commissural interneuron (Fig. 5). This growth cone initially extends towards the ventral midline but turns caudally before reaching the midline. Subsequently the growth cone extends along a caudodorsal pathway before turning into a ventrolateral longitudinal pathway. It is likely that the VeLD growth cones pioneer the ventral longitudinal fasciculus (VLF) since application of diI to the ventral cord backlabels only VeLD neurons in 17-18 hours embryos $(\mathrm{n}=9)$, and the acetylated tubulin antibody labels only VeLD neurons with ventral longitudinal axons in 16-19 hour embryos ( $n=24$; Fig. 6 ). Some VeLD growth cones have extended ten segments by 25 hours of development.

\section{Spatial organization of spinal longitudinal axons}

Application of diI into the dorsal cord of 24-25-hour embryos demonstrated that the longitudinal axons of the $\mathrm{RB}$, DoLA, and ascending commissural neurons are all located in the DLF (Bernhardt et al., '90) and are generally ordered along the dorsal/ventral axis with the RB axons most dorsal and the ascending commissural axons most ventral (data not shown). These observations were reinforced by simultaneously injecting nearby dorsal neurons with LY in 23-30-hour embryos (Fig. 7A). Either nearby RB, DoLA, and contralateral ascending commissural neurons $(n=7)$; RB and DoLA neurons $(n=10)$; $R B$ and 


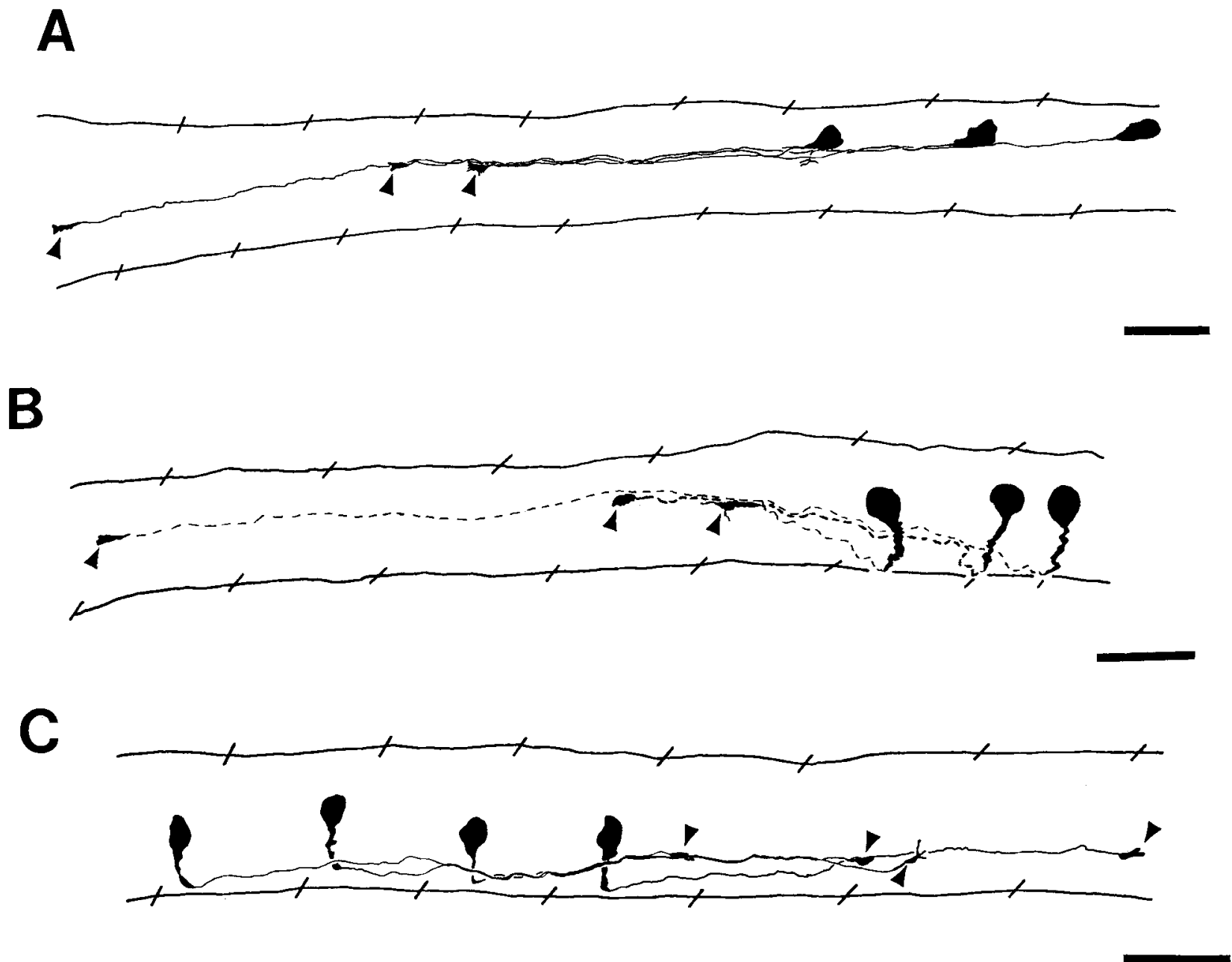

Fig. 8. Growth cones extend in apparent contact with the axons of the same neuronal class. Neurons were labeled with LY/anti-LY. A: Camera lucida drawing of three DoLA neurons from a 22-hour embryo. Arrowheads denote growth cones. Scale, $50 \mu \mathrm{m}$. B: Camera lucida

contralateral ascending commissural neurons $(n=3)$; or DoLA and contralateral ascending commissural neurons $(n=7)$ were filled. Furthermore, labeling axons in 24 -hour embryos with the acetylated tubulin antibody revealed the same general order (Fig. 7B,C).In every case $(n=12)$ the most dorsal and ventral axons in the DLF were $R B$ and ascending commissural axons, respectively. The distributions of these neurons can overlap, however, with RB and DoLA axon pairs intermingling in 8 of the 17 embryos and DoLA and ascending commissural axon pairs intermingling in 3 of 14 embryos assayed with LY injections. In no case, however, did $\mathrm{RB}$ and ascending commissural axon pairs $(\mathbf{n}=10)$ intermingle.

\section{Bundling patterns of homologous longitudinal axons}

The longitudinal axons of a particular neuronal class bundle together and the growth cones of a particular class make apparent contacts with the longitudinal axons of the same class. This was demonstrated in three ways. 1) drawing of three ascending commissural neurons from a 23-hour embryo. Scale, $50 \mu \mathrm{m}$. C: Camera lucida drawing of four VeLD neurons from a 24-hour embryo. Scale, $50 \mu \mathrm{m}$.

Intracellular injections of LY into several neurons of the same class for the DoLA, ascending commissural, and VeLD neurons (Fig. 8) revealed that five of five DoLA growth cones ( $n=5$ embryos), eight of nine ascending commissural growth cones ( $n=5$ embryos), and 13 of 16 VeLD growth cones ( $n=8$ embryos) appeared to contact DoLA axons, ascending commissural axons, and VeLD axons, respectively, in 22-25-hour embryos. 2) DiI orthograde and retrograde labeling of $\mathrm{RB}$, ascending commissural, and VeLD growth cones and axons (Fig. 9A,B) demonstrated that 30 of $31 \mathrm{RB}$ growth cones made apparent contact with $R B$ axons ( $n=12$ embryos; 16-17 hours); five of five ascending commissural growth cones did so with ascending commissural axons ( $\mathrm{n}=5$ embryos; $24-25$ hours); and six of seven ventral longitudinal growth cones at 17-18 hours ( $n=5$ embryos) and five of five growth cones at 24-25 hr $(n=5$ embryos) did so with ventral longitudinal axons. In these embryos RB growth cones were identified by tracing them back to their cell bodies following diI labeling of either the ascending or descending portions of their axons. Growth 

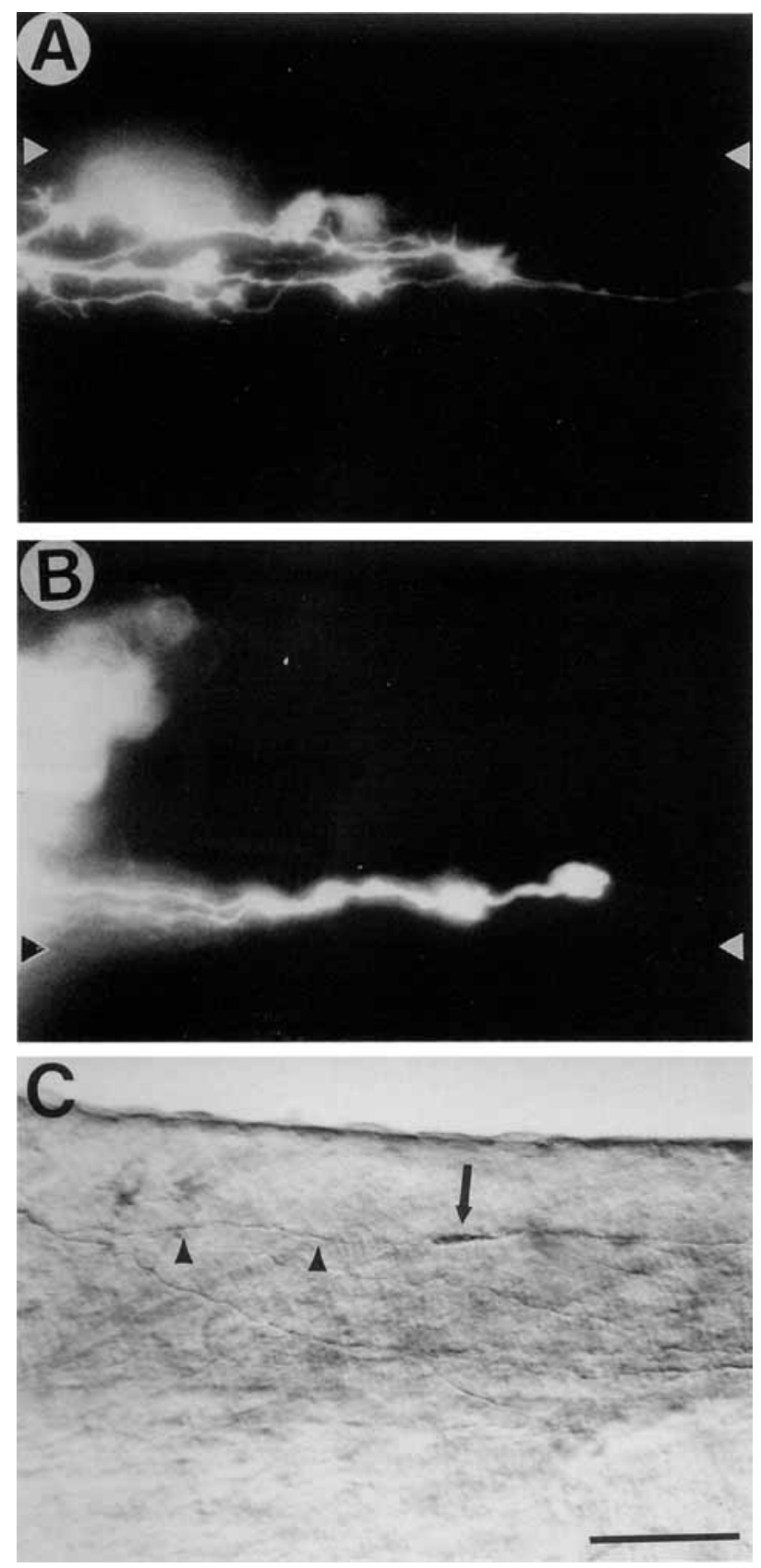

Fig. 9. Growth cones extend in apparent contact with the axons of the same neuronal class. A: Micrograph of diI labeled RB growth cones and axons in a 17-hour embryo. Triangles denote the dorsal border of the cord. Dye application site was to the left of the photograph. B: Micrograph of dil labeled growth cones and axons in the ventral cord of a 17-hour embryo. Application site can be seen to the left. These growth cones and axons probably represent those of VeLD neurons (see text). Triangles denote the ventral border of the cord. C: Micrograph showing an ascending commissural growth cone (arrow) extending on an ascending commissural axon (arrowheads) in a 28-hour embryo which was labeled with anti-CON1 antibody. The rostrodorsally running axons of other ascending commissural neurons can also be seen. The identities of the growth cone and axons was determined by tracing them back to their cell bodies. Scale for A and B, $25 \mu \mathrm{m}$; scale for $\mathrm{C}, 50 \mu \mathrm{m}$ cones were identified as those of ascending commissural neurons by the trajectory of the labeled axons. The ventral longitudinal growth cones and axons were likely VeLD growth cones and axons since anterior to the site of diI injection the only neurons with ventral longitudinal axons backlabeled were the VeLD neurons. 3) Anti-CON1 labeling of ascending commissural neurons $(n=14$ embryos, 28 hours) similarly showed that 14 of 18 ascending commissural growth cones appeared to contact preceding ascending commissural axons (Fig. 9C). In these cases only growth cone/axon pairs in which the growth cone and axon were unambiguously identified by tracing them back to their somata were analyzed. The longitudinal axons of the DoLA and ascending commissural neurons each form relatively tight bundles while the RB and VeLD longitudinal axons each form looser bundles. For the ascending commissural and VeLD neurons bundling correlates with a switch by their growth cones from a circumferential to a longitudinal pathway.

\section{Development of longitudinal spinal tracts}

Sections of cords from 24-hour embryos $(n=3)$ with axons labeled by the acetylated tubulin antibody demonstrated that the longitudinal axons are concentrated in three distinct tracts (DLF, VLF, and MLF; Fig. 10A). Large applications of diI, which covered all or nearly all of the cord in 24-hour embryos $(n=9)$ and cross sections of these cords following photooxidation $(n=3)$ revealed the same distinct tracts (Fig. 10B). In these embryos the DLF contained the RB, DoLA, and ascending commissural axons (Bernhardt et al., '90); the VLF contained the VeLD axons (Bernhardt et al., '90); and the MLF contained the descending axons of the recticulospinal interneurons (Kimmel et al., '82). Furthermore, electron micrographs of sections of the cord in which some VLF and MLF axons were diI labeled and then photooxidized showed that the axons are superficial and that the MLF axons are in the immediate vicinity of the floor plate while the VLF axons are more lateral and dorsal (Fig. 10C). Electron micrographs of the same region in unlabeled embryos at the same stage revealed two bundles of axonal profiles which correspond in position to the VLF and MLF with only a few scattered profiles in between them (Fig. 10D).

The DLF is the first spinal tract to develop, followed shortly by the VLF then later by the MLF and commissural tracts (Table 1). This sequence can be seen in different segments of the same embryo labeled with the acetylated tubulin antibody at 22-24 hours. The anterior segments are more mature and contain the DLF, VLF, and MLF; more posterior segments contain the DLF and VLF; and the most posterior segments contain only the DLF $(n=53$; Fig. 11).

By 30 hours electron micrographs showed that the addition of numerous profiles has created a marginal zone which extends as a continuous band along the lateral margin of the cord (Fig. 12). The marginal zone is largest in the regions of the DLF and VLF and becomes thinner in the intermediate region. Cross-sectioned profiles, which presumably represent dendrites and longitudinal axons, make up the lateral region of the marginal zone and tangentially cut processes of presumably commissural and circumferential axons are found in between the cross-sectioned profiles and neuronal somata. The most dorsal portion of the DLF contains many small caliber axons (approximately $0.25 \mu \mathrm{m}$ 


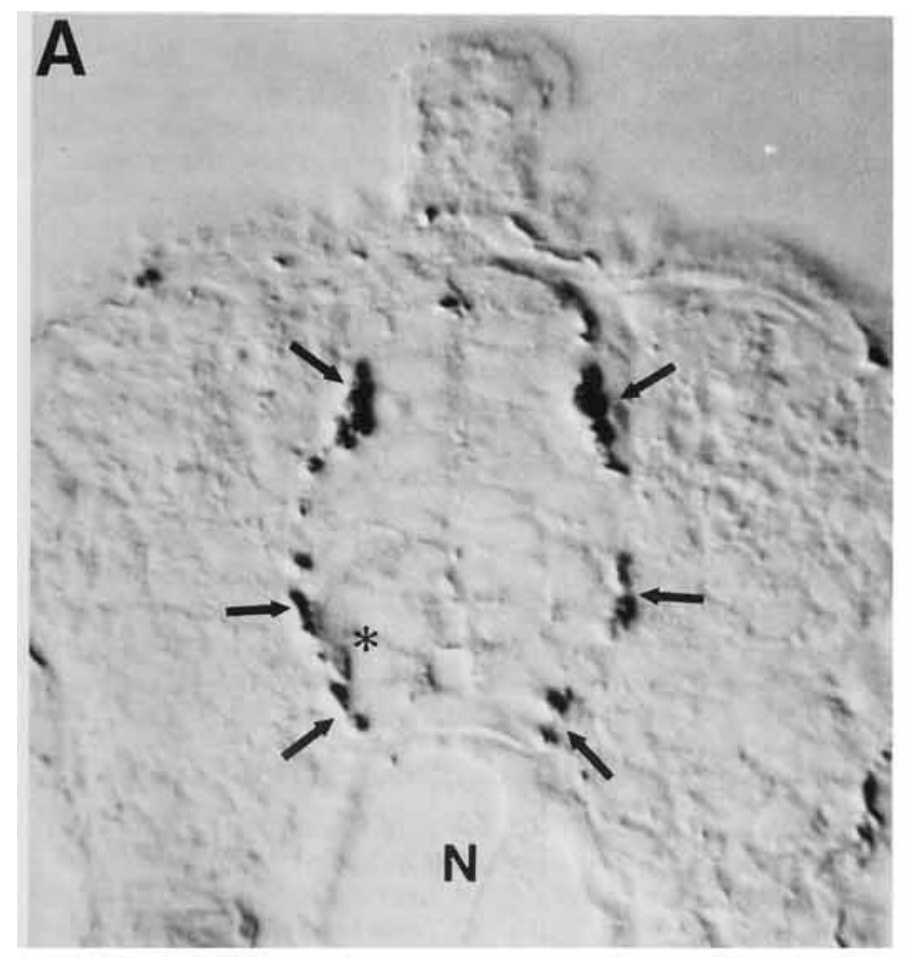

B

\section{C}

3
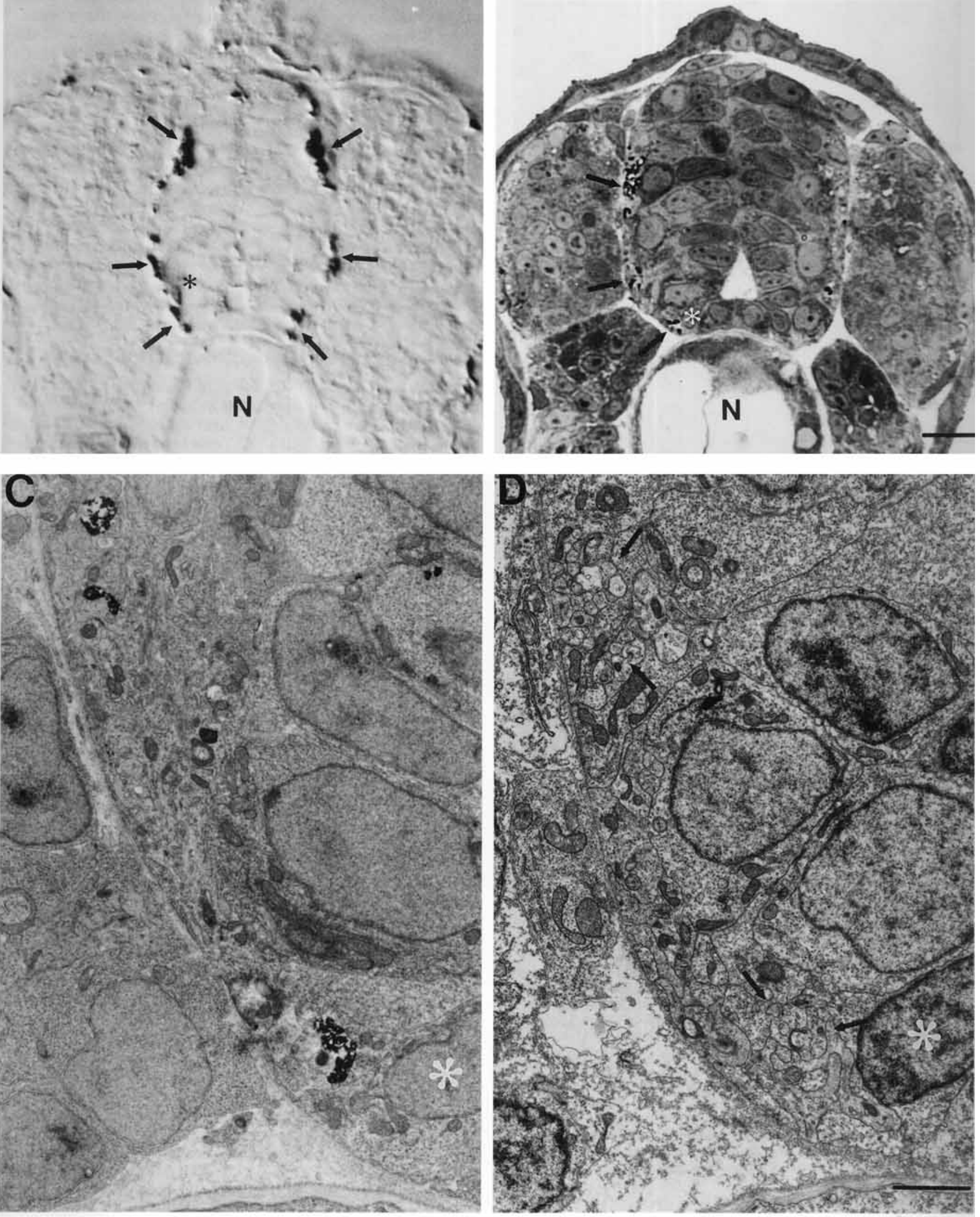

Figure 10 
TABLE 1. Percentage of Embryos in Which Axons Were Present in the DLF, VLF, and MLF, respectively, in S10 and S15 of 16/17-, 18/19-, 24-, and 28-hour Embryos ${ }^{1}$

\begin{tabular}{|c|c|c|c|c|c|c|}
\hline \multirow[b]{2}{*}{ (hours) } & \multicolumn{3}{|c|}{$\mathrm{S} 10$} & \multicolumn{3}{|c|}{$\mathrm{S} 15$} \\
\hline & $\begin{array}{c}\text { DLF } \\
(\%)\end{array}$ & $\underset{(\%)}{\text { VLF }}$ & $\begin{array}{c}\text { MLF } \\
(\%)\end{array}$ & $\begin{array}{c}\text { DLF } \\
(\%)\end{array}$ & $\begin{array}{l}\text { VLF } \\
(\%)\end{array}$ & $\begin{array}{c}\text { MLF } \\
(\%)\end{array}$ \\
\hline $16 / 17$ & 100 & 40 & 0 & 100 & 0 & 0 \\
\hline $18 / 19$ & 100 & 80 & 0 & 100 & 0 & 0 \\
\hline 24 & 100 & 100 & 0 & 100 & 100 & 0 \\
\hline 28 & 100 & 100 & 100 & 100 & 100 & 30 \\
\hline
\end{tabular}

${ }^{1}$ DLF and VLF axons were assayed in embryos ( $n=10$ for each stage) in which axons were labeled by the acetylated tubulin antibody. MLF axons were assayed in embryos ( $n=10$ for each stage) in which axons were labeled with the CON 1 monoclonal antibody. Anti-CON1 recognizes a subset of the earliest neurons in the zebrafish CNS, including many reticulospinal neurons which project into the MLF (Kuwada et al., '87)

in diameter). Ventral to these axons are some of the largest axons in the cord $(0.5-1.0 \mu \mathrm{m}$ in diameter). The small axons may be $\mathrm{RB}$ axons since $\mathrm{RB}$ axons are small and the most dorsal axons (see previous sections). Likewise, the largest axons may be the longitudinal axons of early differentiating ascending commissural neurons since they are amongst the largest spinal axons and are located in the ventral DLF (see previous sections).

\section{Development of ventral commissural tracts}

Electron micrographs show that the first commissural axons insert between the base of the floor plate cells and the well-developed basal lamina, which separates the cord from the underlying notochord (Fig. 13A). At 40 hours, sagittal sections through the midline of the cord reveal numerous profiles, which are presumably commissural axons, arranged in distinct tracts separated by floor plate cells near the ventral midline (Fig. 13B,C). These tracts are numerous and are not segmentally distributed. The tracts of commissural axons were confirmed by labeling axons with the acetylated tubulin antibody in wholemounted larval cords (Fig. 13D).

\section{DISCUSSION \\ Identification of embryonic spinal neurons}

The description of the earliest stages of axonogenesis of spinal neurons depends on the correct identification of very immature neurons. This was done on the basis of the location of the cell body, number of growth cones projected, direction of initial growth cone outgrowth, and time of growth cone projection. For example, the RB, DoLA, and ascending commissural neurons are the only neurons in the early dorsal cord. These cells are readily distinguished since $\mathrm{RB}$ projects two longitudinal growth cones, DoLA a single anterior growth cone, and the ascending commissural neuron a single ventral growth cone. The early ventral neurons are the primary motor neurons $(\mathrm{RoP}, \mathrm{MiP}$, and CaP; Myers et al., '86) and VeLD neuron. The CaP motor neuron cannot be mistaken for VeLD since its growth cone directly leaves the cord from its cell body. Likewise, the RoP and MiP motor neurons cannot be confused with VeLD neurons. Although their somata are located in the same region as the VeLD neurons and they initially extend growth cones posteriorly for up to $1 / 2$ segment before exiting the cord (Myers et al., '86), they differ from the VeLD neuron in the following ways. 1) Although the VeLD neuron is located in a similar region of the cord along the anterior/posterior axis, its cell body is dorsal to those of the motor neurons (see Fig. 11 of Bernhardt et al., '90). 2) MiP and RoP growth cones exit the cord at 18-19 and 20-21 hours, respectively (Pike and Eisen, '90), after extending $1 / 4$ to $1 / 2$ segment within the cord. By 17 hours VeLD growth cones typically have extended $1 \frac{1}{2}$ segments. Assuming that these neurons extend at similar rates, it is likely that the VeLD neuron projects its growth cone several hours before MiP and RoP. 3) The VeLD growth cone initially projects ventrally, while the RoP and MiP growth cones extend laterally from their cell bodies. 4) When VeLD neurons are clearly identifiable on the basis of their axonal trajectory, the trajectory traces out a pathway identical to that followed by the VeLD growth cone.

\section{Stereotyped pathfinding by identified spinal growth cones}

Each segment of the early cord of zebrafish embryos contains a small number of identified neurons (Bernhardt et al., '90) and floor plate cells (Kuwada et al., '90). At 15-17 hours of development the earliest differentiating neurons (RB, DoLA, VeLD, and ascending commissural neurons) project the appropriate number of growth cones, which follow cell specific pathways to reach their normal termination sites in the CNS. This is similar to pathfinding by the three identified primary motor neurons in the zebrafish cord (Eisen et al., '86), the neurons of the nucleus of the posterior commissure in the zebrafish brain (Chitnis and Kuwada, '90), spinal neurons in the Japanese medaka fish embryos (Kuwada, '86), and spinal neurons in Xenopus embryos (Jacobson and Huang, '85; Dale et al., '87). In none of these cases have collateral exuberant axons been observed. In the mammalian CNS some neurons project exuberant axons early only to retract them later (Cowan et al., '84).

\section{Spatial and temporal order of longitudinal spinal axons}

The longitudinal axons in the embryonic cord are spatially ordered. From dorsal to medioventral they are the RB, DoLA, ascending commissural, VeLD, and MLF axons. The RB axons are the first longitudinal axons in the cord and pioneer the DLF. Shortly thereafter, the axons of the VeLD neurons pioneer the VLF. Next are the axons of the DoLA cells followed by the longitudinal axons of the early differentiating ascending commissural neurons. Later the MLF axons invade the spinal cord. In the Xenopus cord the
Fig. 10. At 24-hour the cord contains three discrete longitudinal tracts. A: Cross section of the cord from S8 in which axons were labeled with the acetylated tubulin antibody. From dorsal to ventral the axons are in the DLF, VLF, and MLF, respectively (arrows). Axons seen between the tracts are the circumferential axons of commissural neurons. Also labeled is the initial axonal segment of a ventrally located neuron (asterisk). B: Cross section of the cord from S8 in which axons (arrows) in the DLF, VLF, and MLF were labeled on the left side with diI and then marked with HRP by photooxidization. Some axons were also labeled on the right side. The asterisk provides a reference point which is also indicated in $\mathrm{C}$ and $\mathrm{D}$. N, notochord; scale for A and B, 10 $\mu \mathrm{m}$. C: Electron micrograph of a cross section of the ventrolateral cord from S8 showing two of the axons in the VLF and one of the axons in the MLF in the ventral cord. Axons were labeled with dil followed by photooxidation. D: Electron micrograph of a corresponding cross section of an unlabeled cord showing that there are two distinct bundles of axonal profiles (arrows) with only a few scattered profiles in between them. The two bundles correspond in position to the labeled axons in the VLF and the MLF seen in C. Scale for C and D, $2 \mu \mathrm{m}$. 

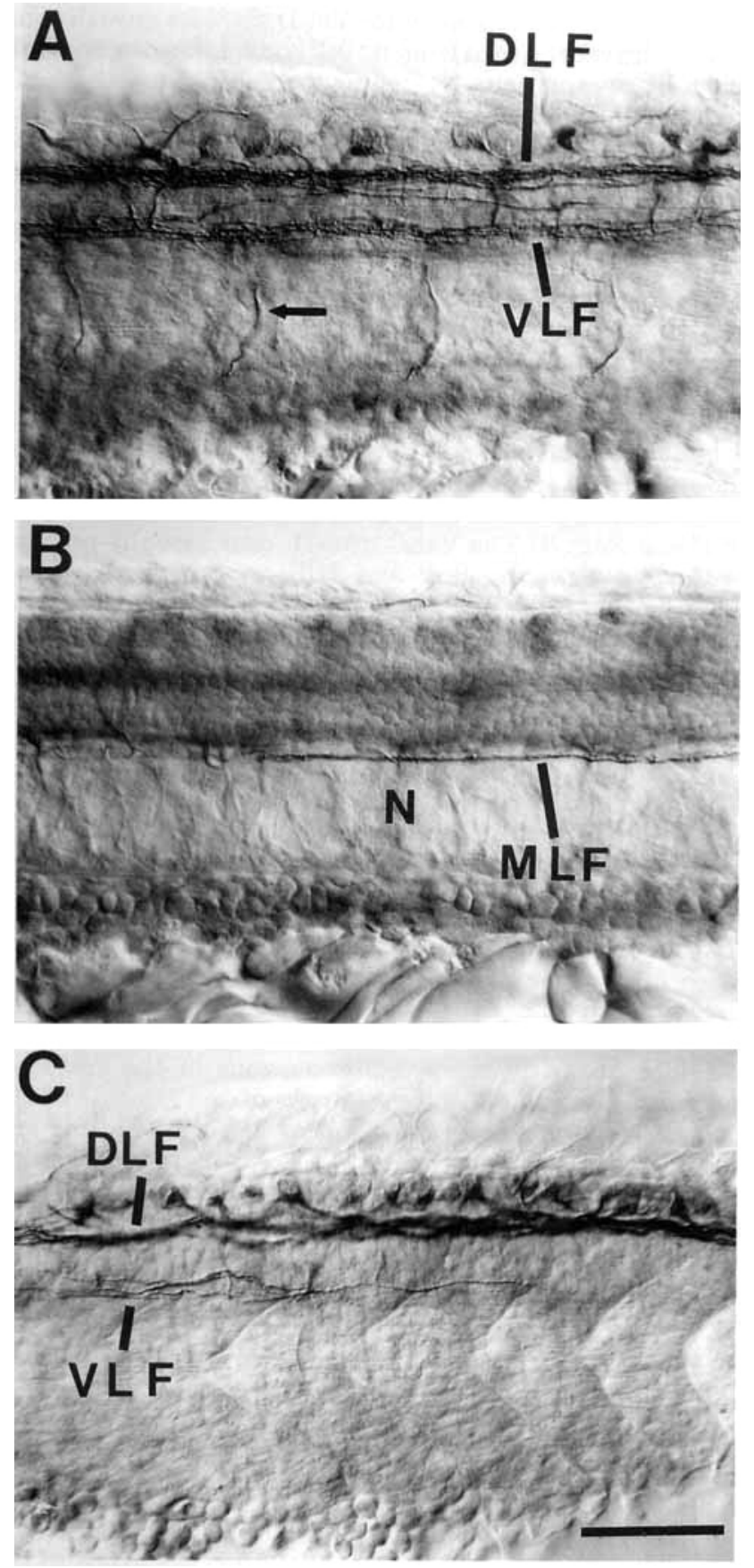

Fig. 11. Temporal order of spinal tract formation. Micrographs from a 24-hour embryo in which all axons were labeled with the acetylated tubulin antibody. A: Photomontage of a midtrunk level spinal cord showing the DLF and VLF. Arrow denotes one of the segmental motor nerves. Labeled RB cell bodies can be seen dorsal to the DLF. B: Same field as A except at a more medial plane of focus to show the MLF. The DLF and VLF can be seen out of the focal plane and dorsal to the MLF. N, notochord. C: More posterior level of the same spinal cord. The DLF runs through these segments but the VLF is found only in the more anterior segments and the MLF is not present at all. Scale for A, B, and C, $50 \mu \mathrm{m}$.

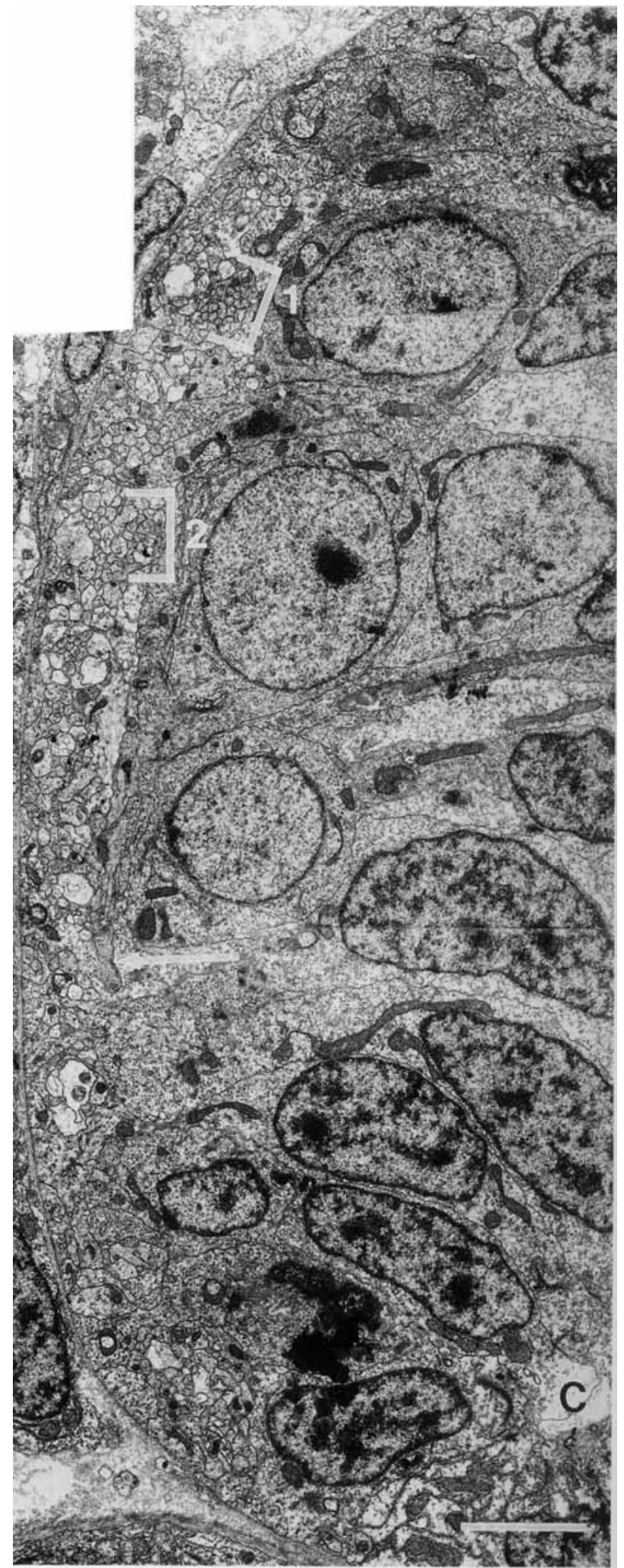

Fig. 12. EM micrograph of a cross section through the 30-hour cord showing that the marginal zone extends from the DLF to the MLF. A dorsal bundle of smaller profiles (1) are probably RB axons and the larger axons (2) ventral to them are probably ascending commissural axons (see text). A circumferentially running axon (arrow) is located medially in the marginal zone. $C$, central canal; scale, $4 \mu \mathrm{m}$. 

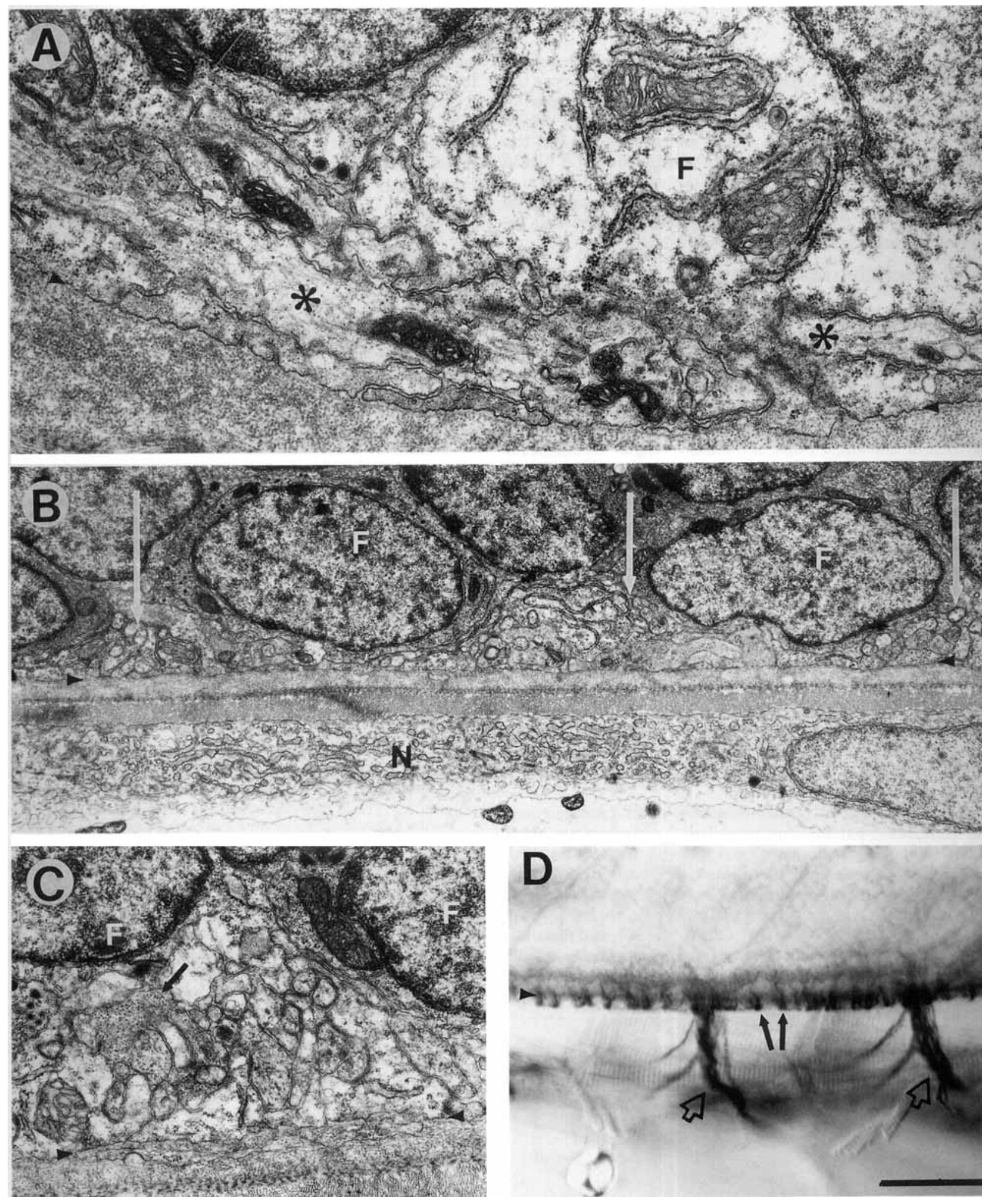

Fig. 13. Discrete spinal commissural tracts. A: EM micrograph of a cross section through the ventral floor region of a spinal cord from a 30-hour embryo. Commissural axons (asterisks) are inserted between the base of a floor plate cell $(\mathrm{F})$ and the ventral basal lamina (arrowheads). Ventral to the cord is a dense matrix which separates the spinal cord from the notochord. B: A sagittal section through the floor region of the cord from a 40-hour embryo. Bundles of commissural axons (arrows) are located between adjacent floor plate cells $(F)$. Cell ventral to the spinal cord is a notochord $(\mathrm{N})$ cell. $\mathrm{C}$ : A high-magnification view

of one bundle of commissural axons seen in a sagittal section of a 40-hour embryo. A large profile (arrow) containing numerous intermediate filaments is probably an early ascending commissural axon (see text). D: Micrograph showing a side view of commissural tracts labeled with the acetylated tubulin antibody in a wholemounted 3-day larva. Two of the commissural tracts are denoted by arrows. Arrowheads, ventral floor of the cord; open arrows, motor nerves. Scale for $A, B, C$, and $D$ are $6,2,4$, and $50 \mu \mathrm{m}$, respectively. 
longitudinal axons also appear in a predictable sequence (Nordlander, '84). In both fish and amphibian embryos the timing of the longitudinal axons rules out a strict spatiotemporal mechanism, in which the first axons are dorsal with each successive axon being more ventral, for the observed spatial order. However, within the DLF timed outgrowth of axons cannot be ruled out since the location of the RB, DoLA, and ascending commissural longitudinal axons along the dorsal/ventral axis and the temporal sequence of their axons are roughly correlated.

\section{Relative attractiveness of growth cone substrates}

The longitudinal axons belonging to one class of spinal neurons bundle together, and their growth cones make apparent contact with the longitudinal axons of the same class. The tendency of longitudinal axons of one neuronal type to bundle can be accounted for by two mechanisms. 1) Affinity of growth cones for the longitudinal axons of neurons of the same class. 2) Affinity of growth cones for some other longitudinally distributed cue. One possibility is the longitudinally arrayed endfeet processes of neuroepithelial cells found along a specified dorsal/ventral level in the cord (Kuwada, '86). At present we cannot distinguish between these two possibilities.

The circumferential growth cones of commissural neurons may display variable affinities to different substrates. The earliest growth cones are relatively far apart and, therefore, do not fasciculate with each other in their circumferential pathways. These growth cones extend on nonaxonal substrates, including the endfeet of neuroepithelial cells and neuronal cell bodies (unpublished data). Commissural growth cones projected at later stages find circumferential axons in their local environment. These growth cones may prefer the axonal substrate (Kuwada et al., '90) and as a consequence lead to the formation of commissural tracts.

The description of pathfinding by spinal growth cones has eliminated pathfinding mechanisms involving a strict spatio-temporal mechanism operating in the whole cord, and the projection and elimination of exuberant axons. Evaluation of other mechanisms requires identification of the different cells and structures which make up the pathways of growth cones (Kuwada et al., ' 90 ), and manipulation of these elements. Owing to the simplicity of the spinal cord of zebrafish embryos this should be achievable.

\section{ACKNOWLEDGMENTS}

We thank G. Piperno for the monoclonal antibody against acetylated tubulin, A. Chitnis for preparing some of the acetylated tubulin labeled embryos, P. Knafl and B. Morin for technical assistance, and $\mathrm{R}$. Hume for critically reading the manuscript. This work was supported by grants from NIH (NS 24848), Office of the Vice-President for Research at the University of Michigan, and a Basil O'Connor Scholar Award from the March of Dimes Birth Defects Foundation to JYK, and a fellowship from the Swiss National Science Foundation to RRB.

\section{LITERATURE CITED}

Bernhardt, R.R., A.B. Chitnis, L. Lindamer, and J.Y. Kuwada (1990) Identification of spinal neurons in the embryonic and larval zebrafish. J. Comp. Neurol. 303:603-616.

Chitnis, A.B., and J.Y. Kuwada (1990) Axonogenesis by identified neurons in the brain of zebrafish embryos. J. Neurosci. 10:1892-1905.

Cowan, W.M., J.W. Fawcett, D.D.M. O'Leary, and B.B. Stanfield (1984) Regressive events in neurogenesis. Science 225:1258-1265.

Dale, N., A. Roberts, O.P. Ottersen, and J. Storm-Mathisen (1987) The development of a population of spinal cord neurons and their axonal projections revealed by GABA immunocytochemistry in frog embryos. Proc. R. Soc. Lond. B. 232:205-215.

Eisen, J.S., P.Z. Myers, and M. Westerfield (1986) Pathway selection by growth cones of identified motoneurons in live zebrafish embryo. Nature 320:269-271.

Jacobson, M., and S. Huang (1985) Neurite outgrowth traced by means of horseradish peroxidase inherited from neuronal ancestral cells in frog embryos. Dev. Biol. 110:102-113.

Kimmel, C.B., S.L. Powell, and W.K. Metealfe (1982) Brain neurons which project to the spinal cord in young larvae of the zebrafish. J. Comp. Neurol. 205:112-127.

Kuwada, J.Y. (1986) Cell recognition by neuronal growth cones in a simple vertebrate embryo. Science 233:740-746.

Kuwada, J.Y., A.B. Chitnis, and L.A. Lindamer (1987) Stereotyped pathfinding by identified growth cones of the embryonic cord. Soc. Neurosci. Abst. 13:1220.

Kuwada, J.Y., R.R. Bernhardt, and A.B. Chitnis (1990) Pathfinding by identified growth cones in the zebrafish spinal cord. J. Neurosci. 10:1229-1308.

Maranto, A.R. (1982) Neuronal mapping: a photooxidation reaction makes Lucifer Yellow useful for electron microscopy. Science 217:953-955.

Myers, P.Z., J. Eisen, and M. Westerfield (1986) Development and axonal outgrowth of identified motoneurons in the zebrafish. J. Neurosci. 6:2278-2289.

Nordlander, R.H. (1984) Developing descending neurons of the caudal spinal cord of early Xenopus. J. Comp. Neurol. 228:117-128.

Pike, S.H., and J.S. Eisen (1990) Identified primary motoneurons in embryonic zebrafish select appropriate pathways in the absence of other primary motoneurons. J. Neurosci. 10:44-49.

Piperno, G., and M.T. Fuller (1985) Monoclonal antibodies specific for an acetylated form of $\alpha$-tubulin recognize the antigen in cilia and flagella from a variety of organisms. J. Cell Biol. 101:2085 - 2094.

Sandell, J.H., and R.H. Masland (1988) Photoconversion of some fluorescent markers to a diaminobenzidene reaction product. J. Histochem. Cytochem. 36:555-559. 